\title{
Dynamical simulations of an electronically induced solid-solid phase transformation in tungsten
}

\author{
Samuel T. Murphy, ${ }^{1, *}$ Szymon L. Daraszewicz, ${ }^{1}$ Yvelin Giret, ${ }^{1}$ Matthew Watkins, ${ }^{1,2}$ Alexander L. Shluger, ${ }^{1}$ \\ Katsumi Tanimura, ${ }^{3}$ and Dorothy M. Duffy ${ }^{1}$ \\ ${ }^{1}$ Department of Physics and Astronomy, University College London, Gower Street, WC1E 6BT, London, United Kingdom \\ ${ }^{2}$ School of Mathematics and Physics, University of Lincoln, Brayford Pool, Lincoln LN6 7TS, United Kingdom \\ ${ }^{3}$ Research Center for Ultra-High Voltage Electron Microscopy, Osaka University, Mihogaoka 8-1, Ibaraki, Osaka 567-0047, Japan
}

(Received 19 May 2015; revised manuscript received 3 August 2015; published 15 October 2015)

\begin{abstract}
The rearrangement of a material's electron density during laser irradiation leads to modified nonthermal forces on the atoms that may lead to coherent atomic motions and structural phase transformation on very short time scales. We present $a b$ initio molecular dynamics simulations of a martensitic solid-solid phase transformation in tungsten under conditions of strong electronic excitation. The transformation is ultrafast, taking just over a picosecond, and follows the tetragonal Bain path. To examine whether a solid-solid bcc-fcc phase transformation could occur during laser irradiation, we use two-temperature molecular dynamics (2T-MD) simulations with a specially developed potential dependent on the electronic temperature. Our simulations show that the occurrence of the solid-solid phase transformation is in competition with ultrafast nonthermally assisted melting with the strength of the electron-phonon coupling determining the lifetime of the new solid phase. In tungsten the melting transition is predicted to occur too rapidly for the fcc phase to be detectable during laser irradiation.
\end{abstract}

DOI: 10.1103/PhysRevB.92.134110

PACS number(s): 34.20.Cf, 64.60.Ej, 64.70.K-, 71.15.Qe

\section{INTRODUCTION}

Structural phase transitions in materials induced by femtosecond laser pulses are of great interest from a fundamental physics perspective, as well as for a number of applications. Ultrafast irradiation of a metallic sample promotes electrons from their ground state into some excited states. Following absorption of the laser energy, the electronic energy distribution tends towards a Fermi-Dirac distribution, defined by an electronic temperature $T_{e}$ through electron-electron collisions. The rate of electron thermalization in metals is rapid (10-100 fs) relative to the rate at which energy is transferred to the ions via electron-phonon coupling [1]. Therefore the system enters a transient, nonequilibrium state where $T_{e} \gg T_{i}$ (where $T_{i}$ is the ionic temperature), sometimes referred to as either a cold plasma state or warm dense matter. The electron density can be significantly modified in a laser-excited state and could dramatically alter the interatomic forces, which could in turn lead to coherent atomic motion (a precursor of solid-solid phase transition), depending on the microscopic interaction for a given material. Such modified interactions, called "nonthermal forces," can induce structural transitions, such as ultrafast nonthermal melting [2-4] or solid-solid phase transformations [5-12], on a very short time scale. As energy is gradually transferred to the ions, the ionic temperature increases and can exceed the material's melting temperature, leading to ultrafast thermal melting.

The majority of metallic elements exhibit either one of the closed packed structures (fcc or hcp) or the more open bcc structure. One may expect that the close-packed structures would be very stable configurations, as each atom is surrounded by 12 neighbors which represents the densest possible packing of hard spheres. However, many metals, such as tungsten, are exceptionally stable in the bcc structure,

*samuel.murphy@ucl.ac.uk while the close-packed fcc and hcp structures are dynamically unstable $[13,14]$. By contrast, for metals where the stable structure is close packed, the bcc structure tends to be dynamically unstable. External constraints, such as pressure, can stabilize the dynamically unstable phases to a point where they become the thermodynamically most stable phase, even if the original phase remains dynamically stable [15]. Under these conditions a nondiffusive, or martensitic, solid-solid phase transformation will occur at a rate determined by the energy barrier along the transitional path.

Recent $a b$ initio calculations by Giret $e t$ al. show that at high electronic temperatures an increase in the electron density in the Cartesian directions can lead to bcc $\mathrm{W}$ becoming dynamically unstable, while both close-packed structures become dynamically stable [12]. As the initial bcc phase becomes unstable there is no barrier to transition to either the fcc phase via the tetragonal Bain path or the hcp phase via the hexagonal Burgers path; therefore a transition could occur on a subpicosecond time scale. While these calculations indicate that a phase transformation is likely, the dynamics of such a phase transformation have not been studied. In particular, it is not clear whether bcc tungsten will transform into the fcc or hcp structure or whether such a transition will occur in the conditions present during ultrafast laser irradiation. Such short time scales should render the structural transformation amenable to study using ab initio molecular dynamics (MD) simulations. Therefore the first aim of this work is to study the dynamics occurring in $\mathrm{W}$ at elevated electronic temperatures using ab initio MD.

Within the Born-Oppenheimer approximation, employed in $a b$ initio MD simulations, the electronic temperature remains constant. As a consequence, energy transfer to the ions via electron-phonon coupling is neglected in these simulations. While it is possible to incorporate electron-phonon coupling into ab initio simulations using such techniques as correlated electron-ion dynamics (CEID) [16] or surface hopping [17,18], these are computationally intensive and cannot be extended 
to supercell sizes sufficient to enable comparisons with time-resolved x-ray or electron diffraction data. As a result, there has been much effort to develop less expensive models based on classical MD where the forces on the ions are determined using an empirical pair potential. Electronic effects may be incorporated by coupling the MD supercell to an electronic subsystem, creating a hybrid continuum-atomistic two-temperature molecular dynamics (2T-MD) model where energy is able to transfer between the two subsystems $[19,20]$. In general these simulations are performed using an empirical potential that has been fitted to reproduce properties of the ground-state system and therefore neglect nonthermal forces. However, this limitation may be, at least partially, ameliorated through the development of electronic temperature-dependent potentials. Daraszewicz et al. have shown that incorporating nonthermal effects via a $T_{e}$-dependent potential makes it possible to reproduce the experimentally observed Bragg peak evolution in laser-irradiated gold nanofilms [20]. Therefore the second aim of this work is to develop a $T_{e}$-dependent potential for $\mathrm{W}$ for use in 2T-MD simulations. Finally, we use these new empirical potentials to examine the possibility of observing a laser-induced solid-solid phase transformation in $\mathrm{W}$ during laser irradiation using 2T-MD.

\section{BORN-OPPENHEIMER MOLECULAR DYNAMICS}

Density functional theory (DFT) MD simulations were performed on a $6 \times 6 \times 6$ supercell, containing $432-\mathrm{W}$ atoms in the $\mathrm{CP} 2 \mathrm{~K}$ code [21]. Exchange correlation was approximated using the generalized gradient approximation (GGA) formulation of Perdew, Burke, and Ernzerhof [22]. W was represented using a mixed Gaussian/plane-wave basis set where the Gaussian basis sets employed a double- $\zeta$ basis set with polarization functions [23]. The plane-wave cutoff for the Goedecker-Teter-Hutter (GTH) pseudopotentials was set to $4762 \mathrm{eV}$ (350 Ry) [24]. Dynamical simulations were performed using a time step of 2.5 fs with the canonical sampling/velocity rescaling (CSVR) $[25,26]$ thermo and barostats and a target temperature of $300 \mathrm{~K}$ and $0 \mathrm{GPa}$ for all elements of the stress tensor. The electronic temperature was set to $20000 \mathrm{~K}$ using the finite temperature generalization to DFT of Mermin [27].

At the beginning of the simulation the $a, b$, and $c$ supercell lattice parameters increase at the same rate (see Fig. 1) as a result of the depopulation of the half-filled $5 d$ and completely filled $6 s$ and $4 f$ bands. As the simulation proceeds, the $a$ lattice parameter increases dramatically while there are small decreases in $b$ and $c$. The oscillations, seen in Fig. 1, are due to the elastic response of the lattice to the sudden introduction of a more repulsive potential. Damping of these oscillations is caused by the action of the thermostat removing both thermal and nonthermal kinetic energy from the system. As the ratios between $a$ and $b$ and $c$ increase, the cell undergoes a martensitic phase transformation along the tetragonal Bain path into the fcc phase, with a stacking fault due to two layers of atoms having a local coordination representative of the hcp phase. As a result of the stacking fault the $a / c$ and $a / b$ ratios reach 1.27 and 1.34, respectively, and the $b / c$ ratio is 1.06 , as opposed to the $\sqrt{2}$, $\sqrt{2}$, and 1 expected from an idealized transformation along the tetragonal Bain path. In order to anneal out the stacking

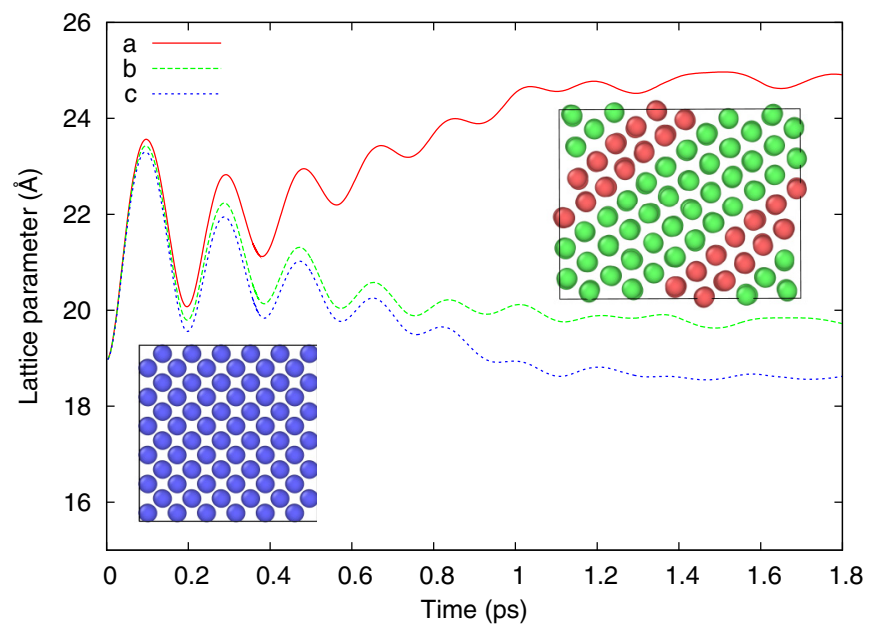

FIG. 1. (Color online) Evolution of the lattice parameters of a 432-atom W supercell during a DFT-MD simulation with $T_{e}=20000 \mathrm{~K}$. The insets show the simulation supercell at the beginning of the simulation and at 1.5 ps. Atoms are colored according to their local coordination, where blue, green, and red spheres represent bcc, fcc, and hcp coordinated atoms, respectively. Identification of coordination environments was performed using the adaptive common neighbor algorithm of Stukowski [28].

fault, further dynamics simulations with a lattice temperature of $600 \mathrm{~K}$ were performed; however, the stacking fault remained on the time scale accessible to DFT-MD.

At $1.8 \mathrm{ps}$ the electronic temperature was instantaneously reduced to $300 \mathrm{~K}$. When $T_{e}$ is reduced, the lattice parameters return to their initial values as the system undergoes a similar martensitic transformation back to the bcc phase within $0.2 \mathrm{ps}$.

Having established that tungsten will undergo a solid-solid phase transition on a picosecond time scale at a constant high electronic temperature, we examine whether this phase transformation will occur during a laser irradiation experiment using 2T-MD. In order to incorporate these nonthermal forces into the 2T-MD simulations, we now develop an electronic temperature-dependent potential for tungsten.

\section{DEVELOPMENT OF THE T $_{e}$-DEPENDENT POTENTIAL}

The majority of the potentials developed for W [29-39] adopt the embedded atom model (EAM), where the total energy $U_{\text {tot }}$ is given as

$$
U_{\mathrm{tot}}=\frac{1}{2} \sum_{i \neq j} V\left(r_{i j}\right)-\sum_{i} f\left(\rho_{i}\right),
$$

where $V\left(r_{i j}\right)$ represents the repulsion of the atoms' nuclei and $f\left(\rho_{i}\right)$ is an embedding term that represents the cohesive energy for an effective electron density, $\rho_{i}$. Within the FinnisSinclair model the embedding energy can be expressed as $f\left(\rho_{i}\right)=-A \sqrt{\rho_{i}}$, where $A$ is an empirically derived potential parameter [29]. The effective electron density on an atom $\rho_{i}$ can be calculated as a pairwise sum of contributions from its 
nearest neighbors, that is,

$$
\rho_{i}=\sum_{j \neq i} \phi\left(r_{i j}\right)
$$

The only materials for which interatomic potentials currently include effects of electronic excitation are Au [40], Si [41-43], Mo [44], and W [45]. The potentials for $\mathrm{Au}$ and $\mathrm{Si}$ were created by assuming the functional forms of the potentials remain unchanged at high $T_{e}$. The potential parameters were derived using force matching, and polynomials of different orders are used to show the parametric dependence on $T_{e}$. The potential for $\mathrm{W}$ was based on the Finnis-Sinclair potential with a modified $T_{e}$-dependent embedding term that incorporates changes in the potential energy and entropy due to electronic excitation explicitly [45]. This approach was extended to other bcc metals by Mason [46].

The electronic free energy $A$ may be written

$$
A=\sum_{i} f_{i} \varepsilon_{i}-\frac{1}{2} E_{H}-\int \rho(r) V_{x c} d r+E_{x c}+E_{Z Z}-T_{e} S .
$$

Here the first term, which is a sum over one-electron eigenenergies $\varepsilon_{i}$, is the band energy (where $f_{i}$ is the Fermi-Dirac occupation numbers for a given $T_{e}$ ). The second term corrects for the double counting of the electron-electron interaction energy in a single-particle treatment. The third term subtracts the energy arising from the exchange correlation potential, and the fourth term adds the correct exchange correlation energy $E_{x c}$. The final two terms are the core-core interaction energy and the contribution of the electronic entropy, respectively. Each of these terms, with the exception of the core-core term, would be expected to have some dependence on the electronic temperature; however, following Khakshouri et al., we assume that this $T_{e}$ dependence is only significant for the band energy and the electronic entropy. In the empirical tight-binding model the remaining terms are treated as a sum of empirical repulsive components. Following this approach these repulsive terms have been attributed to the repulsive part of the EAM potential, i.e.,

$$
\frac{1}{2} \sum_{i j} V\left(r_{i j}\right)=-\frac{1}{2} E_{H}-\int \rho(r) V_{x c} d r+E_{x c}+E_{Z Z} .
$$

As the repulsion is dominated by the core-core term, which, as already mentioned, exhibits no dependency on $T_{e}$, this repulsive term will also show no dependence on the electronic temperature. Consequently, the ground-state repulsive term may be adopted from any of the available empirical potential models. Here we use the extended Finnis-Sinclair potential of Dai et al.[34], as it is capable of reproducing many of the experimentally observed properties of $\mathrm{W}$ while also having a relatively simple functional form. Similarly, the calculation of the effective density is treated using the original potential.

Khakshouri et al.[45] then developed a $T_{e}$-dependent embedding term, based on a rectangular density of states; however, here we adopt a much simpler approach. The $T_{e}$-dependent embedding term is derived by fitting to the free energy-volume surface, calculated from high-temperature DFT, for bcc tungsten. For each volume the effective density is calculated using the original potential and the $T_{e}$-dependent embedding term is then calculated using

$$
\sum_{i} f\left(\rho_{i}, T_{e}\right)=A^{\mathrm{DFT}}\left(\rho_{i}, T_{e}\right)+d E\left(T_{e}\right)-\frac{1}{2} \sum_{i \neq j} V\left(r_{i j}\right),
$$

where $d E\left(T_{e}\right)$ is a constant shift, applied to the DFT free energies at each electronic temperature to ensure that $G=0$ at the limit of the potential for each electronic temperature.

DFT simulations used for fitting the new $T_{e}$-dependent potentials were performed using the ABINIT package $[47,48]$. Exchange correlation was modeled using the local density approximation (LDA). Norm-conserving pseudopotentials that retained the $5 d$ and $6 s$ electrons as valence were employed, and the plane-wave expansion was truncated at $60 \mathrm{Ha}(1632 \mathrm{eV})$. Simulations were performed on the primitive unitcell, containing two atoms, with a $16 \times 16 \times 16$ Monkhorst-Pack $k$-point grid used to sample the Brillouin zone [49]. The calculated lattice parameter of $3.196 \AA$ is in good agreement with the experimental value of $3.16 \AA$, and the phonon spectra are also in excellent agreement with experiment [12]. Free-energy/volume surfaces were generated by performing single-point simulations for a series of volumes for electronic temperatures between 0 and $30000 \mathrm{~K}$ in increments of $5000 \mathrm{~K}$. Potentials for intermediate temperatures were created using cubic splines and stored in tabular form, which can be found in the Supplemental Material [50].

Figure 2(a) shows that as the electronic temperature rises, the free energy decreases due to the increased importance of the entropy $T_{e} S$ contribution, in good agreement with previous results [45,51]. Furthermore, as the electronic temperature increases, the minimum in the free energy is shifted to larger volumes, implying that electronic excitation will cause expansion, as observed in the DFT-MD simulation above. By $30000 \mathrm{~K}$ the minimum in the free energy/volume surface has disappeared and the potential has become completely repulsive.

The new potentials and embedding terms are plotted in Figs. 2(b) and 2(c), respectively. Also included in the plots are the potential and embedding terms for the original Dai potential. The potentials were tested by performing a series of MD simulations at $1 \mathrm{~K}$ within the $N-P-T$ ensemble for electronic temperatures up to $25000 \mathrm{~K}$. The lattice parameters are plotted in Fig. 3. At low electronic temperatures the lattice parameters predicted by the potential are in excellent agreement with those calculated using ABINIT. As $T_{e}$ increases above $11000 \mathrm{~K}$, the $a$ lattice parameter continues to increase; however, there is a small decrease in the $b$ and $c$ lattice parameters, thus breaking the original bcc cubic symmetry, creating a body-centred-tetragonal (bct) phase with space group $14 / M M M$. The $a / c$ ratio continues to increase with increasing electronic temperature until at $17000 \mathrm{~K}$ it reaches $\sqrt{2}$, at which point another phase transformation occurs, this time to the fcc structure. Taking the final relaxed fcc structure at $20000 \mathrm{~K}$ and restarting the simulation with the $T_{e}$ reduced to $300 \mathrm{~K}$ results in a transformation back to the initial bcc structure. These simulations suggest that the potential is capable of reproducing some important aspects of the phonon spectrum, such as the dynamic stabilities of the bcc and fcc phases. 


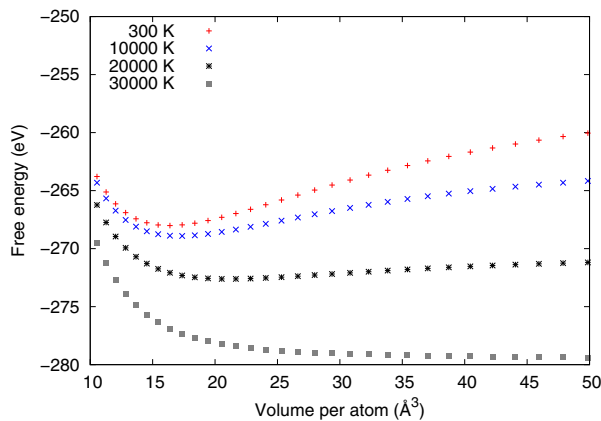

(a) DFT

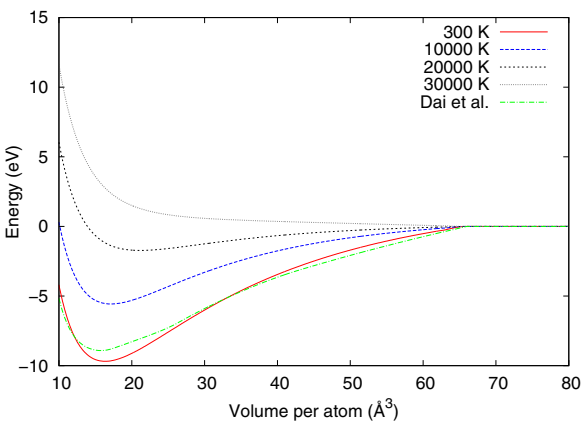

(b) New potentials

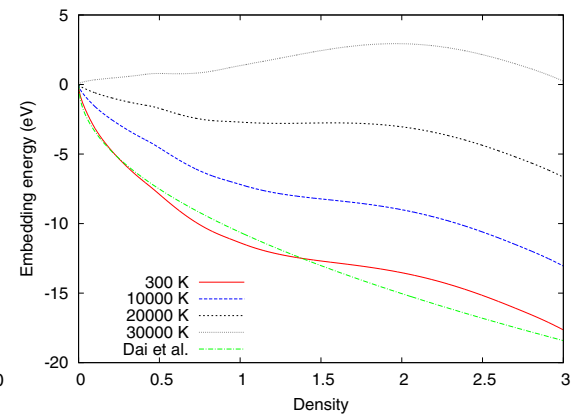

(c) Embedding term

FIG. 2. (Color online) Details of the $T_{e}$-dependent potential. The per atom free energy calculated using DFT that is used to fit the new potential (a). As $T_{e}$ is increased, the electronic entropy drives the free energy lower as well as increasing the equilibrium volume. (b) The energy per atom calculated using the new potentials. (c) The new embedding term.

In order to provide a direct comparison with the DFT simulation presented in Fig. 1, a similar simulation was performed in a cell containing 432 atoms and a lattice temperature of $300 \mathrm{~K}$ using the new potential at $20000 \mathrm{~K}$. The evolution of the lattice parameters is presented in Fig. 4. In contrast to the DFT simulation shown above, the simulation using the empirical potential does not generate a stacking fault. The presence of the stacking fault in the DFT simulation may be due to the choice of barostat and the barostat relaxation time; however, it is not possible to reproduce the exact simulation conditions using the empirical potential because of the different barostats implemented in the codes.

As shown in Fig. 2, the well depth of the potentials decrease as the electronic temperature is increased, implying that the

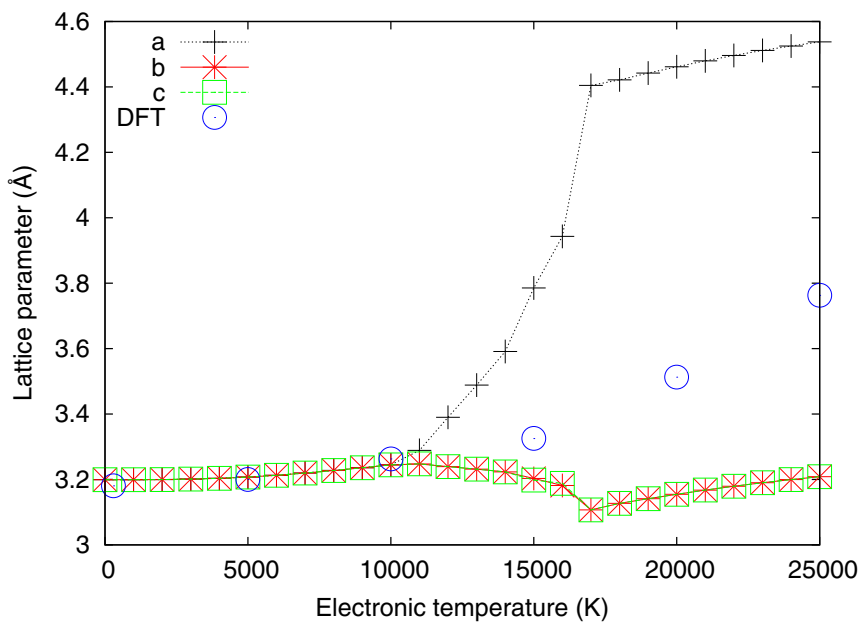

FIG. 3. (Color online) Lattice parameters for tungsten as a function of the electronic temperature $T_{e}$ calculated using the new empirical potential and DFT. DFT lattice parameters were determined by performing a series of single point calculations at increasing volumes, and so are constrained to be bcc. Lattice parameters calculated using the empirical potential are the result of energy minimization at constant pressure. The empirical potential predicts that at low electronic temperatures the bcc phase is stable and the lattice parameters are in good agreement with the DFT; however, at intermediate values of $T_{e}$ the bct phase becomes dominant. At high electronic temperatures the fcc structure becomes dominant. melting temperature would decrease. We examine how the melting point changes as a function of the electronic temperature using the moving interface method [52]. The melting temperature of $\mathrm{W}$ calculated as a function of $T_{e}$ is presented in Fig. 5. In the ground state, the melting point is predicted to be approximately $3800 \mathrm{~K}$, which is in good agreement with the experimental value of $3695 \mathrm{~K}$ [53] and the $3925 \mathrm{~K}$ calculated by Liu et al. using MD and the Dai potential [54]. As $T_{e}$ increases, the melting temperature is shown to decrease dramatically, and it appears that it will reach zero at the point at which the minimum in the potential energy surface disappears completely and the crystal becomes unstable, as would be anticipated. By contrast, Feng et al. predict, based on their vacancy formation energies at high $T_{e}$ calculated using DFT, that the melting temperature of tungsten will increase as the electronic temperature is increased [51]. We note that these simulations were performed in a supercell with a fixed

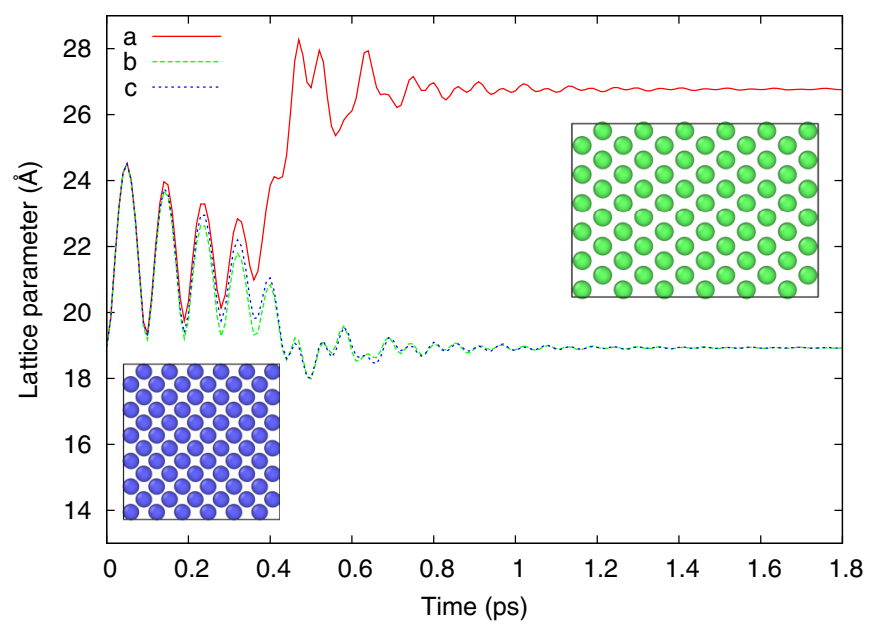

FIG. 4. (Color online) Evolution of the lattice parameters of a 432-atom W supercell during a MD simulation using empirical potentials with $T_{e}=20000 \mathrm{~K}$. The insets show the simulation supercell at the beginning of the simulation and at $1.5 \mathrm{ps}$. Atoms are colored according to their local coordination, where blue and green spheres represent bcc and fcc coordinated atoms, respectively. Identification of coordination environments was performed using the adaptive common neighbor algorithm of Stukowski [28]. 


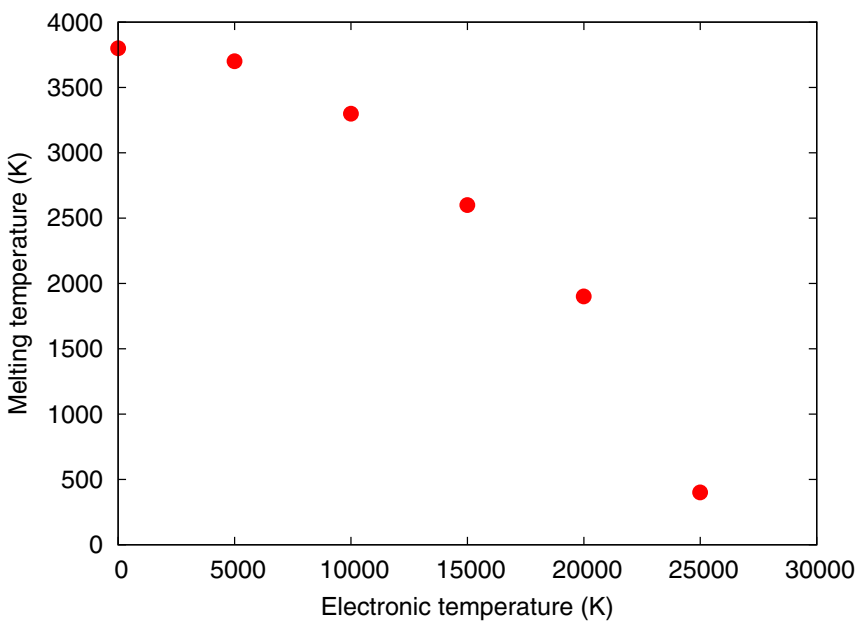

FIG. 5. (Color online) Melting temperature of tungsten as a function of the electronic temperature. We note that at $15000 \mathrm{~K}$ the calculated melting point is for the bct structure. Similarly, at 20000 and $25000 \mathrm{~K}$ the melting point is for fcc W.

volume. A decrease in the melting temperature, similar to that observed here, has been observed in molecular dynamics simulations of Si under constant pressure conditions [42]. This reduction in the melting point is nonthermal and therefore can only be incorporated into the simulation through the use of $T_{e}$-dependent potentials, as employed here.

\section{2T-MD SIMULATIONS OF LASER IRRADIATION OF W THIN FILMS}

In order to investigate the possibility of a phase transformation being observed under conditions typical of a laser irradiation experiment, we employ two-temperature molecular dynamics simulations. Within the 2T-MD method the atoms are represented as an array of points that are allowed to evolve in time using MD with the forces between atoms calculated using the new $T_{e}$-dependent potential. The influence of processes such as electronic stopping and electron-phonon coupling are explicitly incorporated into the ion dynamics by coupling the ion system to a continuum electronic subsystem and allowing energy transfer between the two systems.

The 2T-MD method assumes that the evolution of the electronic temperature in time follows the heat diffusion equation, i.e.,

$$
C_{e}\left(T_{e}\right) \frac{\partial T_{e}}{\partial t}=\nabla \cdot\left(\kappa_{e} \nabla T_{e}\right)-G\left(T_{e}\right)\left(T_{e}-T_{i}\right)+S(z, t),
$$

where $C_{e}\left(T_{e}\right)$ is the electronic specific heat, $G\left(T_{e}\right)$ is the electron-phonon coupling, and $\kappa_{e}$ is the electronic heat conductivity. $S(z, t)$ is a source term that in this case represents energy deposited by the laser. The source term is described as a pulse that is Gaussian in time and has an exponentially decreasing amplitude in $z$, that is,

$$
S(z, t)=\left(\frac{2 F}{l_{p} t_{p}} \frac{\sqrt{\ln 2}}{\pi}\right) e^{-4 \ln 2\left(t-t_{0}\right)^{2} / t_{p}^{2}} e^{-z / l_{p}},
$$

where $F$ is the absorbed fluence, $l_{p}$ is the optical penetration depth of the sample at the wavelength of the pulse length, $t_{p}$ is the duration of the pulse at the FWHM, and $t_{0}$ is time zero, defined as the maximum of the laser pulse on the sample surface [55]. As we are only considering thin films in this study, we can assume homogeneous excitation of the electronic subsystem and so the $\kappa_{e}$ and $z$ dependencies in Eqs. (6) and (7) disappear.

A number of studies have suggested that the electronphonon coupling is significantly enhanced in ultrathin films of typically two atomic layers; however, at thicknesses comparable to those studied here, the electron-phonon coupling is expected to be similar to the bulk value [56,57]. Therefore we use the electronic temperature-dependent electron-phonon coupling and specific heats for bulk W, with $G_{0}$ calculated in previous work [58] and extrapolated to high $T_{e}$ following the methodology of Lin et al. [59]. For low electronic temperatures, such as those encountered here, our calculated electronic specific heats are in excellent agreement with Lin et al. and more recent DFT studies [60-62]. The ionic subsystem is evolved in parallel with the electronic subsystem using classical MD. The traditional equations of motion are modified, such that

$$
m_{i} \frac{\partial \mathbf{v}_{i}}{\partial t}=\mathbf{F}_{i}+\tilde{\mathbf{F}}_{i}\left(T_{t}\right)
$$

where $\mathbf{v}_{i}$ is the velocity of an atom with mass, $m_{i} . \mathbf{F}_{i}$ is the classical force acting on the atom calculated using the empirical potential, and $\tilde{\mathbf{F}}_{i}$ is the additional driving term, based on a modified Langevin thermostat formulation:

$$
\tilde{\mathbf{F}}_{i}\left(T_{t}\right)=-\gamma \mathbf{v}_{i}+\mathbf{f}_{L}\left(T_{t}\right)
$$

where $\gamma$ represents a frictional drag force, $\mathbf{f}_{L}$ is the stochastic force, and $T_{t}$ is the target temperature of the thermostat, which is set equal to $T_{e}$. For more details of the formulation of the Langevin thermostat and its dependence on $G\left(T_{e}\right)$, refer to previous work $[20,63,64]$.

Laser irradiation simulations were performed using a local version of the DL-POLY (4.01) code [65] on a tungsten slab, created by taking $30 \times 30 \times 60$ repetitions of the bcc unit cell, with a resulting slab thickness of $\sim 190 \AA$ containing 108000 atoms. The simulation supercell was periodic in $x$ and $y$, and the supercell length in $z$ was doubled to allow for any possible expansion during laser irradiation, with final supercell lattice parameters of $x=96.18, y=96.18$, and $z=384.72 \AA$. As the area of the laser spot is greater than the simulation cell in $x$ and $y$, we assume that the cell boundaries remain fixed during the simulation, as the excited atoms surrounding the simulation cell impart a sufficient pressure to prohibit expansion in the lateral directions.

The ionic simulation supercell was initially relaxed under constant volume conditions using a Nose-Hoover thermostat at $300 \mathrm{~K}$ for $6 \mathrm{ps}$ with a time step of $0.2 \mathrm{fs}$. Once equilibration was complete, the ionic system was connected to the continuum system and the initial electronic temperature set to $300 \mathrm{~K}$. Energy was deposited into the electronic subsystem by a laser pulse with a FWHM $=76$ fs and an absorbed fluence sufficient to ensure the electronic temperature was high enough to induce 


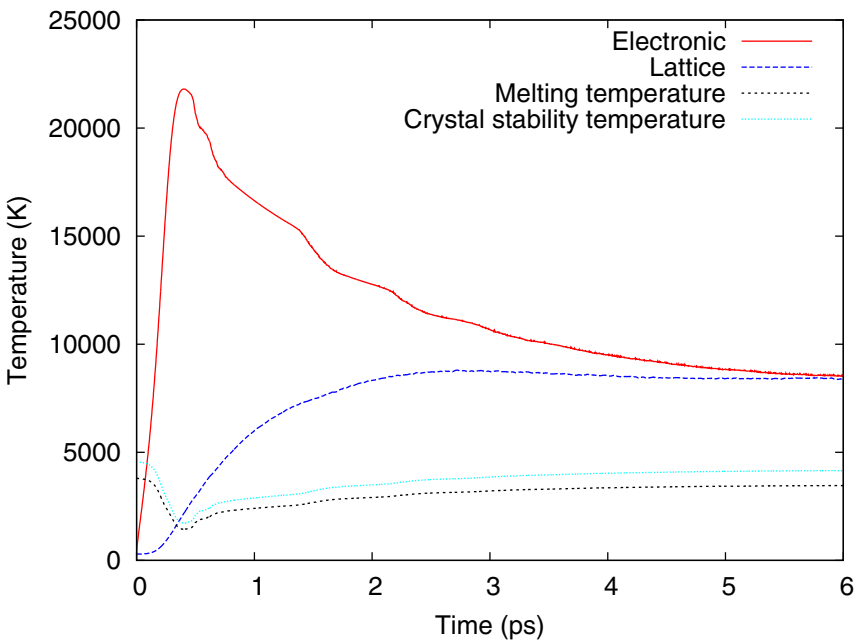

FIG. 6. (Color online) Evolution of the electronic and ionic temperatures during irradiation of tungsten with a 76-fs laser pulse delivering a fluence of $80 \mathrm{~mJ} \mathrm{~cm}^{-2}$. Also included in the plot are the $T_{e}$-dependent melting and crystal stability temperatures.

the phase transformation (in this case $F=80 \mathrm{~mJ} \mathrm{~cm}^{-2}$ and $l_{p}=150 \AA$ ). The system was subsequently allowed to evolve for 6 ps.

Figure 6 shows the evolution of the electronic and ionic temperatures during laser irradiation. During the laser pulse the electronic temperature rapidly increases until reaching a maximum electronic temperature of $\sim 22000 \mathrm{~K}$ at $\sim 0.4$ ps. As energy is transferred to the ionic subsystem, the average lattice temperature climbs rapidly and exceeds the $T_{e}$-dependent melting and crystal stability temperatures within 0.34 and $0.36 \mathrm{ps}$, respectively. Finally, the electronic and ionic temperatures reach equilibrium at $6 \mathrm{ps}$.

Figure 7 shows the atomic scale evolution of the thin film during laser irradiation and is split into two sections. All atoms in the cell, color coded according to the local bonding environment, are shown in Figs. 7(a)-7(e). As the simulation proceeds the film undergoes a homogenous melting process with nearly all traces of bcc tungsten having disappeared by $0.8 \mathrm{ps}$. From these images it appears that there is no solid-solid phase transformation due to the high electronic temperature; however, if we look at atoms with the fcc structure only, as shown in Figs. $7(\mathrm{~g})-7(\mathrm{j})$, we can see that there is a concentration of atoms that are locally fcc coordinated beneath the film's surfaces. In fact, as much as $1 \%$ of all atoms in the system have an fcc coordination at 0.5 ps. However, from this point the concentration of fcc-coordinated atoms begins to decrease. This decrease does not occur due to the electronic temperature dropping below that required to stabilize the fcc phase, which occurs at $0.91 \mathrm{ps,} \mathrm{but} \mathrm{due} \mathrm{to}$ the very rapid increase in the ionic temperature leading to ultrafast non-thermally assisted melting. We refer to this as nonthermally assisted ultrafast melting due to the significant decrease in the melting temperature arising from changes in the empirical potential at elevated electronic temperatures. We note that Wahrenberg et al. have observed the presence of a fcc-like liquid phase on the surface of laser-irradiated tungsten from time-resolved photoelectron spectroscopy [66].

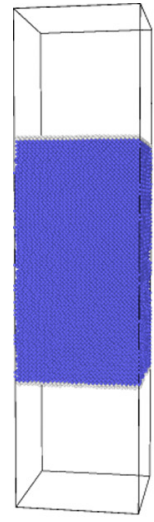

(a) 0 ps

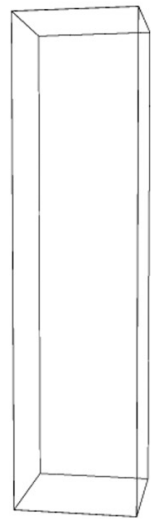

(f) 0 ps

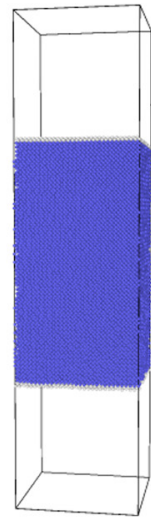

(b) $0.2 \mathrm{ps}$

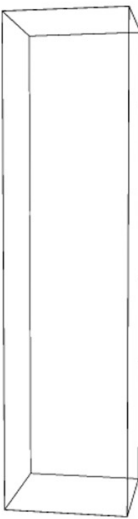

(g) $0.2 \mathrm{ps}$

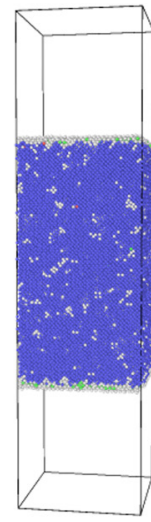

(c) $0.4 \mathrm{ps}$

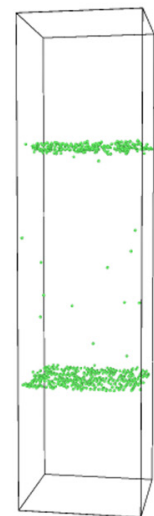

(h) $0.4 \mathrm{ps}$

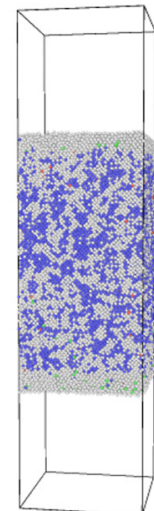

(d) $0.6 \mathrm{ps}$

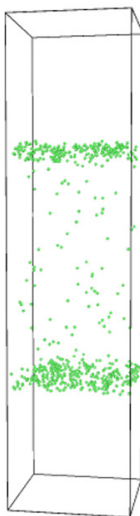

(i) $0.6 \mathrm{ps}$

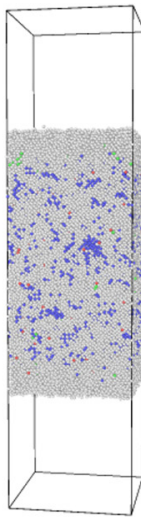

(e) $0.8 \mathrm{ps}$

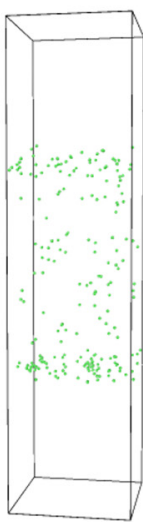

(j) $0.8 \mathrm{ps}$
FIG. 7. (Color online) Structure of the tungsten slab during irradiation with a 76-fs laser pulse of fluence $80 \mathrm{mJcm}^{-2}$. In the diagrams the atoms are colored according to their local structure: blue corresponds to atoms that are locally bcc, green spheres are atoms displaying the fcc structure, and white spheres correspond to atoms which are deemed to have no crystal structure. Local structures were determined using the adaptive common neighbor analysis [28]. All atoms are shown in Figs. 7(a)-7(e), and only atoms that are fcc coordinated are shown in Figs. 7(f)-7(j).

\section{DISCUSSION}

Our simulation suggests that while a small number of atoms modify their local coordination environment, the ultrafast nonthermally assisted melting occurs so rapidly that it would not be possible to observe the solid-solid phase transformation in tungsten experimentally. The speed with which energy is transferred to the ions is due to the material-dependent electron-phonon coupling. For tungsten the electron-phonon coupling is known to be very strong, i.e., an order of magnitude greater than that calculated for gold $[58,59]$. While it may not be possible to observe a solid-solid phase transformation in tungsten, it is possible that other metals may become dynamically unstable due to electronic excitation and have a high enough melting point and low enough electron-phonon coupling to stabilize the new phase. To investigate this scenario we performed a further simulation on a material that has many of the same physical properties as tungsten; however, 


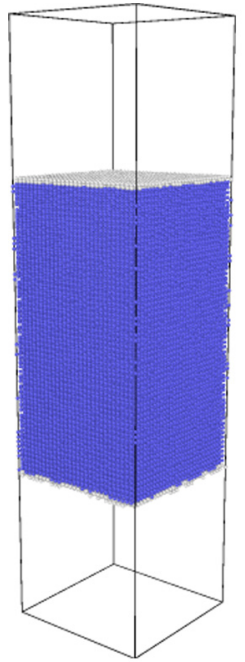

(a) 0 ps

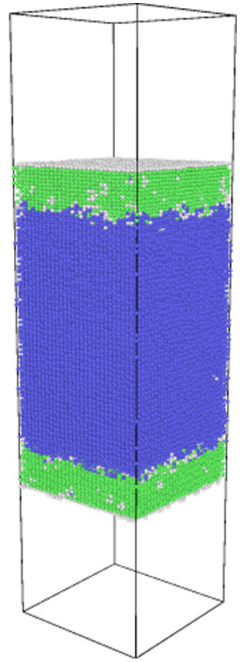

(b) 1 ps

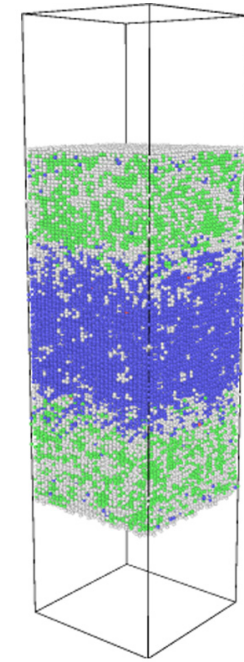

(c) $2 \mathrm{ps}$
FIG. 8. (Color online) Structure of the tungsten slab during irradiation with a 76 -fs laser pulse of fluence $80 \mathrm{mJcm}^{-2}$, where the electron-phonon coupling has been artificially reduced by an order of magnitude. In the diagrams the atoms are colored according to their local structure: blue corresponds to atoms that are locally bcc, green spheres are atoms displaying the fcc structure, and white spheres correspond to atoms which are deemed to have no crystal structure. Local structures were determined using the adaptive common neighbor analysis [28].

the electron-phonon coupling has been reduced by an order of magnitude.

The reduction in the electron-phonon coupling reduces the rate at which energy is transferred to the lattice so the increase in the ionic temperature is much slower, ensuring the film takes longer to melt. Figure 8 shows heterogeneous nucleation of the fcc phase from the film's surfaces. The average velocity of the phase transformation fronts was $2360 \mathrm{~ms}^{-1}$, which is $45 \%$ of the speed of sound in tungsten (taken as $5220 \mathrm{~ms}^{-1}$ ) [67]. This phase front velocity is substantially greater than that expected for the heterogeneous melting transition [68]. Approximately $25 \%$ of the film has undergone a transition to the fcc structure prior to melting. A structural transformation of such a large proportion of the film should be observable using ultrafast $\mathrm{X}$-ray or electron diffraction.

\section{SUMMARY}

In this work we show a dynamical simulation of a solidsolid phase transformation in a metal driven by electronic excitation using DFT assuming a constant electronic temperature. We have developed a $T_{e}$-dependent interatomic potential that is capable of reproducing the dynamical stabilities of the relevant phases as a function of the electron temperature, thereby reproducing this phase transformation. The new potential was then used in 2T-MD simulations that replicate the conditions likely to occur during femtosecond laser irradiation of tungsten thin films. The 2T-MD simulations show that under these conditions ultrafast nonthermally assisted melting dominates over the solid-solid phase transformation. The new potential will enable us to study other experimental configurations that may impede energy transfer to the ions, thereby giving the phase transition sufficient time to occur. The methods applied both for the 2T-MD and fitting of the empirical potential may be applied to a wide variety of other materials which display properties that may be more accommodating for a solid-solid phase transformation.

\section{ACKNOWLEDGMENTS}

S.T.M. acknowledges funding from the Leverhulme trust (Grant No. RPG-2013-331). Via our membership in the UK's HPC Materials Chemistry Consortium, which is funded by EPSRC (EP/L000202), this work made use of the facilities of HECToR and ARCHER, the UK's national high-performance computing service, which is funded by the Office of Science and Technology through EPSRC's High End Computing Programme. Additional computational resources were provided by the high performance computer center at UCL.
[1] K. H. Bennemann, Ultrafast dynamics in solids, J. Phys.: Condens. Matter 16, R995 (2004).

[2] A. Rousse, C. Rischel, S. Fourmaux, I. Uschmann, S. Sebban, G. Grillon, P. Balcou, E. Forster, J. P. Geindre, P. Audebert, J. C. Gauthier, and D. Hulin, Non-thermal melting in semiconductors measured at femtosecond resolution, Nature (London) 410, 65 (2001).

[3] K. Sokolowski-Tinten, C. Blome, C. Dietrich, A. Tarasevitch, M. H. von Hoegen, D. von der Linde, A. Cavalleri, J. Squier, and M. Kammler, Femtosecond X-ray Measurement of Ultrafast Melting and Large Acoustic Transients, Phys. Rev. Lett. 87, 225701 (2001).

[4] P. L. Silvestrelli, A. Alavi, M. Parrinello, and D. Frenkel, Ab Initio Molecular Dynamics Simulation of Laser Melting of Silison, Phys. Rev. Lett. 77, 3149 (1996).

[5] P. Beaud, S. L. Johnson, E. Vorobeva, U. Staub, R. A. DeSouza, C. J. Milne, Q. X. Jia, and G. Ingold, Ultrafast Structural
Phase Transition Driven by Photoinduced Melting of Charge and Orbital Order, Phys. Rev. Lett. 103, 155702 (2009).

[6] J. Kanasaki, E. Inami, K. Tanimura, H. Ohnishi, and K. Nasu, Formation of Sp3-Bonded Carbon Nanostructures by Femtosecond Laser Excitation of Graphite, Phys. Rev. Lett. 102, 087402 (2009).

[7] C. Z. Wang, K. M. Ho, M. D. Shirk, and P. A. Molian, LaserInduced Graphitization on a Diamond (111) Surface, Phys. Rev. Lett. 85, 4092 (2000).

[8] H. O. Jeschke, M. E. Garcia, and K. H. Bennemann, Microsocopic analysis of the laser-induced femtosecond graphitization of diamond, Phys. Rev. B 60, R3701 (1999).

[9] R. Nuske, A. Jurgilaitis, H. Enquist, M. Harb, Y. Fang, U. Hakanson, and J. Larsson, Transforming graphites to nanoscale diamonds by a femtosecond laser pulse, Appl. Phys. Lett. 100, 043102 (2012). 
[10] N. Medvedev, V. Tkachenko, and B. Ziaja, Modelling of nonthermal solid-to-solid phase transition in diamond irradiated with femtosecond x-ray FEL pulse, Contrib. Plasma Phys. 55, 12 (2015).

[11] E. S. Zijlstra, N. Huntemann, and M. E. Garcia, Laser-induced solid-solid phase transition in As under pressure: A theoretical prediction, New J. Phys. 10, 033010 (2008).

[12] Y. Giret, S. L. Daraszewicz, D. M. Duffy, A. L. Shluger, and K. Tanimura, Nonthermal solid-to-solid phase transitions in tungsten, Phys. Rev. B 90, 094103 (2014).

[13] V. Ozolins, First Principles Calculations of Free Energies of Unstable Phases: The Case of Fcc W, Phys. Rev. Lett. 102, 065702 (2009).

[14] K. Einarsdotter, B. Sadigh, G. Grimvall, and V. Ozolins, Phonon Instabilities in Fcc and Bcc Tungsten, Phys. Rev. Lett. 79, 2073 (1997).

[15] G. Grimvall, B. Magyari-Kope, V. Ozolins, and K. A. Persson, Lattice instabilities in metallic elements, Rev. Mod. Phys. 84, 945 (2012)

[16] A. P. Horsfield, M. Finnis, M. Foulkes, J. LePage, D. Mason, C. Rave, A. P. Sutton, D. R. Bowler, A. J. Fisher, R. Miranda, L. Stella, A. M. Stoneham, D. Dundas, E. McEniry, T. N. Todorov, and C. G. Sanchez, Correlated electron-ion dynamics in metallic systems, Comput. Mater. Sci. 44, 16 (2008).

[17] J. C. Tully and R. K. Preston, Trajetory surface hopping approach to nonadiabatic molecular collisions: The reaction of $\mathrm{H}^{+}$with $\mathrm{D}_{2}$, J. Chem. Phys. 55, 562 (1971).

[18] D. S. Scholl and J. C. Tully, A generalised surface hopping method, J. Chem. Phys. 109, 7702 (1998).

[19] D. S. Ivanov and L. V. Zhigilei, Combined atomistic-continuum modeling of short-pulse laser melting and disintegration of metal films, Phys. Rev. B 68, 064114 (2003).

[20] S. L. Daraszewicz, Y. Giret, N. Naruse, Y. Murooka, J. Yang, D. M. Duffy, A. L. Shluger, and K. Tanimura, Structural dynamics of laser-irradiated gold nanofilms, Phys. Rev. B 88, 184101 (2013).

[21] J. Hutter, M. Iannuzzi, F. Schiffmann, and J. VandeVondele, cp2k: atomisitic simulations of condensed matter systems, Wiley Inter. Re.: Comput. Mol. Sci. 4, 15 (2014).

[22] J. P. Perdew, K. Burke, and M. Ernzerhof, Generalized Gradient Approximation Made Simple, Phys. Rev. Lett. 77, 3865 (1996).

[23] G. Lippert, J. Hutter, and M. Parrinello, A hybrid Gaussian and plane wave density functional scheme, Mol. Phys. 92, 477 (1997).

[24] S. Goedecker, M. Teter, and J. Hutter, Seperable dual-space Gaussian pseudopotentials, Phys. Rev. B 54, 1703 (1996).

[25] G. Bussi, D. Donadio, and M. Parrinello, Canonical sampling through velocity rescaling, J. Chem. Phys. 126, 014101 (2007).

[26] G. Bussi, T. Zykova-Timan, and M. Parrinello, Isothermalisobaric molecular dynamics using stochastic velocity rescaling, J. Chem. Phys. 130, 074101 (2009).

[27] N. D. Mermin, Thermal properties of the inhomogeneous electron gas, Phys. Rev. 137, A1441 (1965).

[28] A. Stukowski, Visualisation and analysis of atomistic simulation data with OVITO - The open visualization tool, Modell. Simul. Mater. Sci. Eng. 20, 045021 (2012).

[29] M. W. Finnis and J. E. Sinclair, A simple empirical n-body potential for transition metals, Philos. Mag. 50, 45 (1984).
[30] G. J. Ackland and R. Thetford, An improved n-body semiempirical model for body-centred cubic transition metals, Philos. Mag. 56, 15 (1987).

[31] L. T. Kong, X. Y. Li, W. S. Lai, J. B. Liu, and B. X. Liu, Interfacial reaction of $w / c u$ examined by an n-body potential through molecular dynamics simulations, Japan J. Appl. Phys. 41, 4503 (2002).

[32] R. F. Zhang, H. R. Gong, L. T. Kong, and B. X. Liu, Comparative study of metastable phase formation in the immiscible $\mathrm{Cu}-\mathrm{W}$ system by ab initio calculation and n-body potential, J. Phys.: Condens. Matter 16, 5251 (2004).

[33] R. F. Zhang, Y. X. Shen, H. F. Yan, and B. X. Liu, Formation of amorphous alloys by ion beam mixing and its multiscale theoretical modeling in the equilibrium immiscible $\mathrm{Sc}-\mathrm{W}$ system, J. Phys. Chem. B 109, 4391 (2005).

[34] X. D. Dai, Y. Kong, J. H. Li, and B. X. Liu, Extended FinnisSinclair potential for bcc and fcc metals and alloys, J. Phys. Condens. Matter 18, 4527 (2006).

[35] P. M. Derlet, D. Nguyen-Manh, and S. L. Dudarev, Multiscale modeling of crowdion and vacancy defects in body-centeredcubic transition metals, Phys. Rev. B 76, 054107 (2007).

[36] C. Bjorkas, K. Nordlund, and S. L. Dudarev, Modelling radiation effects using the $\mathrm{ab}$ initio based tungsten and vanadium potentials, Nucl. Instrum. Methods Phys. Res. B 267, 3204 (2009).

[37] N. Juslin and B. D. Wirth, Interatomic potentials for simulation of the bubble formation in W, J. Nucl. Mater. 432, 61 (2013).

[38] M.-C. Marinica, L. Wentelon, M. R. Gilbert, L. Proville, S. L. Dudarev, J. Marian, G. Bencteux, and F. Willaime, Interatomic potentials for modeling radiation defects and dislocations in tungsten, J. Phys.: Condens. Matter 25, 395502 (2013).

[39] J. Wang, Y. L. Zhou, M. Li, and Q. Hou, A modified W-W interatomic potential based on ab initio calculations, Modelling Simul. Mater. Sci. Eng. 22, 015004 (2014).

[40] G. E. Norman, S. V. Starikov, and V. V. Stegailov, Atomistic simulation of laser ablation of gold: Effect of pressure relaxation, J. Exp. Theor. Phys. 114, 792 (2012).

[41] L. Shokeen and P. K. Schelling, An empirical potential for silicon under conditions of strong electronic excitation, Appl. Phys. Lett. 97, 151907 (2010).

[42] L. Shokeen and P. K. Schelling, Thermodynamics and kinetics of silicon under conditions of strong electronic excitation, J. Appl. Phys. 109, 073503 (2011).

[43] L. Shokeen and P. K. Schelling, Role of electronic-excitation effects in the melting and ablation of laser excited silicon, Comput. Mater. Sci. 67, 316 (2013).

[44] J. A. Moriarty, R. Q. Hood, and L. H. Yang, QuantumMechanical Interatomic Potentials with Electron Temperature for Strong-Coupling Transition Metals, Phys. Rev. Lett. 108, 036401 (2012).

[45] S. Khakshouri, D. Alfe, and D. M. Duffy, Development of an electron-temperature-dependent interatomic potential for molecular dynamics simulation of tungsten under electronic excitation, Phys. Rev. B 78, 224304 (2008).

[46] D. Mason, Incorporating non-adiabatic effects in embedded atom potentials for radiation damage cascade simulations, J. Phys.: Condens. Matter 27, 145401 (2015).

[47] X. Gonze, B. Amadon, P.-M. Anglade, J.-M. Beuken, F. Bottin, P. Boulanger, F. Bruneval, D. Caliste, R. Caracas, M. Côté, T. Deutsch, L. Genovese, Ph. Ghosez, M. Giantomassi, S. 
Goedecker, D. R. Hamann, P. Hermet, k, F. Jollet, G. Jomard, S. Leroux, M. Mancini, S. Mazevet, M. J. T. Oliveira, G. Onida, Y. Pouillon, T. Rangel, G.-M. Rignanese, D. Sangalli, R. Shaltaf, M. Torrent, M. J. Verstraete, G. Zerah, and J. W. Zwanziger, ABINIT: First-principles approach to material and nanosystem properties, Comput. Phys. Commun. 180, 2582 (2009).

[48] X. Gonze, G.-M. Rignanese, M. Versraete, J.-M. Beuken, Y. Pouillon, R. Caracas, F. Jollet, M. Torrent, G. Zerah, A. Mikami, P. Ghosez, M. Veithen, J.-Y. Raty, V. Olevano, F. Bruneval, L. Reining, R. Godby, G. Onida, D. R. Hamann, and D. C. Allan, A brief introduction to the ABINIT software package, Z. Kristallogr. 220, 558 (2005).

[49] H. J. Monkhorst and J. D. Pack, Special points for Brillouin-zone integrations, Phys. Rev. B 13, 5188 (1976).

[50] See Supplemental Material at http://link.aps.org/supplemental/ 10.1103/PhysRevB.92.134110 for the $\mathrm{T}_{e}$-dependent potentials, tabulated for the DL_ POLY code.

[51] S. Feng, J. Zhao, X. Cheng, and H. Zhang, Theoretical calculation on electronic excitation and compression effect in tungsten, Physica B 413, 69 (2013).

[52] J. F. Lutsko, D. Wolf, S. R. Phillpot, and S. Yip, Molecular dynamics study of lattice-defect-nucleated melting in metals using an embedded-atom-method potential, Phys. Rev. B 40, 2841 (1989).

[53] D. Lide, Handbook of Chemistry and Physics, 81st ed. (CRC Press, Boca Raton, FL, 2000-2001).

[54] C.-M. Liu, X.-R. Chen, C. Xe, L.-C. Cai, and F.-Q. Jing, Melting curves and entropy of fusion of body-centered cubic tungsten under pressure, J. Appl. Phys. 112, 013518 (2012).

[55] Y. Giret, A. Gelle, and B. Arnaud, Entropy Driven Atomic Motion in Laser-Excited Bismuth, Phys. Rev. Lett. 106, 155503 (2011).

[56] T. Valla, M. Kralj, M. Milun, P. Pervan, P. D. Johnson, and Woodruff, Oscillatory electron-phonon coupling in ultra-thin silver films on V(100), J. Phys.: Condens. Matter 12, L477 (2000).

[57] W. Ma, H. Wang, X. Zhang, and W. Wang, Study of the electron-phonon relaxation in thin metal films using transient thermoreflectance technique, Int. J. Thermophys. 34, 2400 (2013).

[58] S. L. Daraszewicz, Y. Giret, H. Tanimura, D. M. Duffy, A. L. Shluger, and K. Tanimura, Determination of the electron-phonon coupling constant in tungsten, Appl. Phys. Lett. 105, 023112 (2014).

[59] Z. Lin, L. V. Zhigilei, and V. Celli, Electron-phonon coupling and electron heat capacities of metals under conditions of strong electron-phonon nonequilibrium, Phys. Rev. B 77, 075133 (2008).

[60] G. V. Sin'ko, N. A. Smirnov, A. A. Ovechkin, P. R. Levashov, and K. V. Khishchenko, Thermodynamic functions of the heated electron subsystem in the field of cold nuclei, High Energy Density Phys. 9, 309 (2013).

[61] E. Bevillon, J. P. Colombier, V. Recoules, and R. Stoian, Free electron propereties of metals under ultrafast laser-induced electron-phonon non-equilibriums: A first principles study, Phys. Rev. B 89, 115117 (2014).

[62] E. Bevillon, J. P. Colombier, V. Recoules, and R. Stoian, First principles calculations of heat capacities of ultrafast laserexcited electrons in metals, Appl. Surf. Sci. 336, 79 (2015).

[63] D. M. Duffy and A. M. Rutherford, Including the effects of electronic stopping and electron-ion interactions in radiation damage simulations, J. Phys.: Condens. Matter 19, 016207 (2007).

[64] A. M. Rutherford and D. M. Duffy, The effect of electron-ion interactions on radiation damage simulations, J. Phys.: Condens. Matter 19, 496201 (2007).

[65] I. T. Todorov, S. W. K. Trachenko, and M. T. Dove, DLPOLY3: New dimensions in molecular dynamics simulations via massive parallelism, J. Mater. Chem. 16, 1911 (2006).

[66] R. Wahrenberg, H. Stupp, H.-G. Boyen, and P. Oelhafen, Electronic structure of liquid tungsten studied by time-resolved photoelectron spectroscopy, Europhys. Lett. 49, 782 (2000).

[67] Edited by D. R. Lide, CRC Handbook of Chemistry and Physics, 7th ed. (CRC Press, Boca Raton, FL, 1991).

[68] D. S. Ivanov and L. V. Zhigilei, Kinetic Limit of Heterogeneous Melting in Metals, Phys. Rev. Lett. 98, 195701 (2007). 